\title{
Scheme for preparation of mulipartite entanglement of atomic ensembles
}

\author{
Peng Xue*, and Guang-Can Guo ${ }^{\dagger}$ \\ Key Laboratory of Quantum Information, University of Science and \\ Technology \\ of China, Hefei 230026, P. R. China
}

We describe an experimental scheme of preparing multipartite $\mathrm{W}$ class of maximally entangled states between many atomic ensembles. The scheme is based on laser manipulation of atomic ensembles and single-photon detection, and well fits the status of the current experimental technology. In addition, we show one of the applications of the kind of W class states, teleporting an entangled state of atomic ensembles with unknown coefficients to more than one distant parties, either one of which equally likely receives the transmitted state.

PACS number(s): 03.67.-a, 03.65.Ud, 42.50.Gy

Quantum entanglement is one of the most striking features of quantum mechanics. The recent surge of interest and progress in quantum information theory allows one to take a more positive view of entanglement and regard it as an essential resource for many ingenious applications such as quantum computation [1-3], quantum teleportation [4,5], superdense coding [6], and quantum cryptography [7-9]. The technology of generation and manipulation of bipartite entangled states has been realized in some systems [10-13]. Recently, there has been much interest in using quantum resource to get more and more subsystems entangled [14-17] for more useful applications $[18,19]$. In most of the above schemes, the subsystems are taken as single-particle. Remarkably, Lukin and Duan et al. have proposed some schemes [20-23] for preparation of entanglement which use atomic ensembles with a large number of identical atoms as the basic system. For example, one can use atomic ensembles for generation of substantial spin squeezing [24] and continuous variable entanglement [21,25], and for efficient preparation of Einstein-Podolsky-Rosen (EPR) [22] and Greenberger-Horne-Zeilinger (GHZ) type of maximally entangled states [23]. The schemes have some special advantages compared with other quantum information schemes based on the control of single particles [26]. However, there is not any scheme for experimental realization of $\mathrm{W}$ class states in this system.

It is well known that there are two different kinds of genuine tripartite entanglement_ - GHZ state and W state [27]. Indeed, any (non-trivial) tripartite entangled state can be converted, by means of stochastic local op-

\footnotetext{
*Email address: xuepeng@mail.ustc.edu.cn

${ }^{\dagger}$ Email address: gcguo@ustc.edu.cn
}

erations and classical communication (SLOCC), into one of two standard forms, namely either the GHZ state or else the W state, and that this splits the set of the genuinely trifold entangled states into two sets which are unrelated under local operations and classical communication (LOCC). That is, the W state can not be obtained from a GHZ state by means of LOCC and thus one could expect, in principle that it has some interesting, characteristic properties. The entanglement of the $\mathrm{W}$ class state is maximally robust under disposal of any one of three qubits, in the sense that the remaining reduced density matrices retain, according to several criteria, the greatest possible amount of entanglement, compared to any other state of three qubits, either pure or mixed. So it is important to prepare the $\mathrm{W}$ class of entangled state experimentally.

In this report, we describe an experimental scheme of preparing multipartite $\mathrm{W}$ class of maximal entanglement between atomic ensembles. The scheme involves laser manipulation of atomic ensembles, beam splitters, and single-photon detection, and well fits the status of the current experimental technology. The first step of this scheme is to entangle two atomic ensembles in an EPR state, which is based on the techniques proposed in Ref. [22]. To prepare the $\mathrm{W}$ class of maximally entangled states, two laser pulses (pumping laser and repumping laser) are applied to the atomic ensembles and the corresponding Raman transition $|g\rangle \rightarrow|s\rangle$ and anti-Raman transition $|s\rangle \rightarrow|g\rangle$ occur for several times. In addition, we show one of the applications of the kind of $\mathrm{W}$ class states, teleporting an entangled state of atomic ensembles with unknown coefficients to many distant parties, either one of which equally likely receives the transmitted state.

Let us have a look at the generalized form $\left|W_{M}\right\rangle$ of the $\mathrm{W}$ class state in multi-qubit systems. In Ref. [27], the state is defined as

$$
\left|W_{M}\right\rangle=(1 / \sqrt{M})|M-1,1\rangle,
$$

where $|M-1,1\rangle$ denotes the totally symmetric state including $M-1$ zeros and 1 one. For example, we obtain $M=3$,

$$
\left|W_{3}\right\rangle=(1 / \sqrt{3})(|001\rangle+|010\rangle+|100\rangle) .
$$

The basic element of this scheme is an ensemble of many identical alkali atoms with a Raman type $\Lambda$-level 
configuration coulped by a pair of optical fields with the Rabi frequencies $\Omega$ and $\omega$, respectively, shown as Fig. 1, the experimental realization of which can be either a room-temperature dilute atomic gas $[25,28]$ and a sample of cold trapped atoms $[29,30]$. We continue to use the symbols and corresponding definitions shown in Refs. [22,23]. A pair of metastable lower states $|g\rangle$ and $|s\rangle$ can be achieved, for example, in hyperfine or Zeeman sublevels of electronic ground states of alkali atoms. The atoms in the ensembles are initially prepared to the ground state $|g\rangle$ through optical pumping. The transition $|g\rangle \rightarrow|e\rangle$ is coupled by the classical laser with the Rabi frequency $\Omega$ and the forward scattering Stokes light comes from the transition $|e\rangle \rightarrow|s\rangle$ [22]. The pumping laser is shined on all atoms so that each atom has an equal small probability to be excited into the state $|s\rangle$ through the Raman transition. After the atomic gas interacts with a weak pumping laser, there will be a special atomic mode $s$ called the symmetric collective atomic mode

$$
s=\left(1 / \sqrt{N_{a}}\right) \sum_{i=1}^{N_{a}}|g\rangle_{i}\langle s|,
$$

where $N_{a} \gg 1$ is the total atom number. In particular, an emission of the single Stokes photon in a forward direction results in the state of atomic ensembles given by $s^{+}|v a c\rangle$, where the ensemble ground state $|v a c\rangle=\otimes_{i}|g\rangle_{i}$. The scheme for preparation of $\mathrm{W}$ class of maximally entangled states between atomic ensembles works in the following way (seeing Fig 2):

1 The first step is to share an EPR type of entangled state between two distant ensembles 1 and 2 using the scheme shown in Ref. [22]. The ensembles are illuminated by a weak pumping laser pulse which couples resonantly the transition $|g\rangle \rightarrow|e\rangle$ and we look at the spontaneous emission light from the transition $|e\rangle \rightarrow|s\rangle$, whose frequency is assumed to be different to the pumping laser. There are two pulses with the frequencies $\omega_{\text {pump }}$ and $\omega_{\text {repump }}$, respectively, which correspond to the pumping and repumping process. Here two pumping laser pulses excite both ensembles simultaneously and with probability $p_{c}$ the projected state of the ensembles 1 and 2 is an EPR state with the form

$$
|\psi\rangle_{12}=\left(s_{1}^{+}+e^{i \phi_{12}} s_{2}^{+}\right) / \sqrt{2}|v a c\rangle_{12},
$$

where $\phi_{12}=\phi_{2}-\phi_{1}$ is a difference of the phase shift which is fixed by the optical channel connecting the two ensembles, and $|v a c\rangle_{12}$ denotes that both ensembles are in the ground state $|g\rangle$.

2 We then connect the other two distant ensembles 2 and 3. Since the ensemble 3 is prepared to the ground state $|g\rangle$, the whole system is described by the state $|\psi\rangle_{12} \otimes|v a c\rangle_{3}$. Here two pumping pulses excite both ensembles simultaneously and the forward scattering Stokes light from both ensembles is combined at the $50 / 50$ beam splitter (BS) after some filters which filter out the pumping laser pulses with the outputs detected by the two single-photon detectors D1 and D2, respectively. If one photon is detected by either of the detectors, we obtain the state

$$
|\psi\rangle_{123}=\left(s_{2}^{+}+e^{i \phi_{23}} s_{3}^{+}\right) / \sqrt{2}|\psi\rangle_{12} \otimes|v a c\rangle_{3} .
$$

Otherwise, we need to manipulate repumping pulses to the transition $|s\rangle \rightarrow|e\rangle$ on the three ensembles and set them back to the ground state. Then, we repeat the steps 1 and 2 until finally we obtain a click in either of the two detectors.

3 A repumping laser pulse with the frequency $\omega_{\text {repump }}$ is applied to ensemble 2. If one excitation is registered from it, we succeed and go on with the next step. Otherwise, we need to repeat the above steps until we get the three ensembles in the entangled state $\left|W^{\prime}\right\rangle_{123}$ successfully.

$$
\begin{aligned}
\left|W^{\prime}\right\rangle_{123} & =s_{2}\left(s_{2}^{+}+e^{i \phi_{23}} s_{3}^{+}\right)\left(s_{1}^{+}+e^{i \phi_{12}} s_{2}^{+}\right)|v a c\rangle_{123} \\
& =\left(s_{1}^{+}+2 e^{i \phi_{12}} s_{2}^{+}+e^{i \phi_{13}} s_{3}^{+}\right)|v a c\rangle_{123},
\end{aligned}
$$

where $s_{2} s_{2}^{+} s_{2}^{+}|v a c\rangle_{2}=2 \frac{N_{a}-1}{N_{a}} s_{2}^{+}|v a c\rangle_{2} \simeq 2 s_{2}^{+}|v a c\rangle_{2}$ $\left(N_{a} \gg 1\right)$.

4 However, it is evident that the state $\left|W^{\prime}\right\rangle_{123}$ above does not belong to the $\mathrm{W}$ class of maximally entangled states shown in Eqs. (1) and (2). Then we connect the ensembles 1 and 3 using the same way in the step 2, and apply a repumping laser pulse to the ensemble 1 after a click in D4 or D5. If there is one excitation is registered by $\mathrm{D} 6$, we obtain the $\mathrm{W}$ class of maximally entangled state

$$
\begin{aligned}
|W\rangle_{123} & =s_{1}\left(s_{1}^{+}+e^{i \phi_{13}} s_{3}^{+}\right) / 2 \sqrt{3}\left|W^{\prime}\right\rangle_{123} \\
& =\left(s_{1}^{+}+e^{i \phi_{12}} s_{2}^{+}+e^{i \phi_{13}} s_{3}^{+}\right) / \sqrt{3}|v a c\rangle_{123} .
\end{aligned}
$$

5 Similarly, suppose that the ensembles 1 and 2 are in the EPR state $|\psi\rangle_{12}$, to entangle $n$ ensembles in the W class state, firstly we connect the ensembles $i$ and $i+1$, and then repump the ensemble $i$ ( $i$ from 2 to $n-1$ ) after a right click orderly. It needs to repeat the steps 2 and 3 for $n-2$ times to obtain the $n$-party $\mathrm{W}$ class of nonmaximally entangled state

$$
\begin{aligned}
\left|W^{\prime}\right\rangle_{1 \ldots n} & =\prod_{i=2}^{n-1} s_{i}\left(s_{i}^{+}+e^{i \phi_{i, i+1}} s_{i+1}^{+}\right)\left(s_{1}^{+}+e^{i \phi_{12}} s_{2}^{+}\right)|v a c\rangle_{1 \ldots n} \\
& =\left(s_{1}^{+}+2 \sum_{i=2}^{n-1} e^{i \phi_{1 i}} s_{i}^{+}+e^{i \phi_{1 n}} s_{n}^{+}\right)|v a c\rangle_{1 \ldots n}
\end{aligned}
$$

The difference of phase shift $\phi_{1 i}$ is fixed by the possible asymmetry of the setup and in principle can be measured. So we can put some suitable phase shifters with relative phase shift to counteract it. Then we need to repeat the 
manipulation above to the ensembles 1 and $n$. Thus, we can entangle $n$ ensembles in the W class of maximally entangled states

$$
\begin{aligned}
|W\rangle_{1 \ldots n} & =\frac{1}{2 \sqrt{n}} s_{1}\left(s_{1}^{+}+e^{i \phi_{1 n}} s_{n}^{+}\right)\left|W^{\prime}\right\rangle_{1 \ldots n} \\
& =\frac{1}{\sqrt{n}} \sum_{i=1}^{n} e^{i \phi_{1 i}} s_{i}^{+}|v a c\rangle_{1 \ldots n} .
\end{aligned}
$$

Note $\left|W^{\prime}\right\rangle$ in Eqs. (6) and (8) are not normalized. The normalization constant for Eq. (8) is $1 / \sqrt{4 n-6}$.

Now, we consider the efficiency of this scheme, which is usually described by the total generation time. Since the probability for getting a click of either of two detectors is given by $p_{c}$, we entangle $n$ ensembles in the $\mathrm{W}$ class state with the probability $\left(p_{c}\right)^{n}$. In the generation process, the dominant noise is the photon loss, which includes the contributions from the channel attenuation, the spontaneous emissions in the atomic ensembles, the coupling inefficiency of Stokes light into and out of the channel, and the inefficiency of the single-photon detectors which can no perfectly distinguish between one and two photons. All the above noise is described by an overall loss probability $\eta$. Due to the noise, the total generation time is represented by $T \sim t_{0} /\left[(1-\eta)^{2 n-1} p_{c}^{n}\right]$, where $t_{0}$ is the light-atom interaction time. And the generation time increases with the number of ensembles exponentially by the factor $1 /\left[(1-\eta)^{2} p_{c}\right]$.

Also with the noise, the state of the ensembles is actually described by

$$
\rho_{n}=\frac{1}{c_{n}+1}\left(c_{n} \rho_{v a c}+|W\rangle_{1 \ldots n}\langle W|\right),
$$

where the vacuum coefficient $c_{n}$ is basically given by the conditional probability for the inherent modemismatching noise contribution (please seeing Ref. [26] for details) and $\rho_{v a c}$ stands for the vacuum component with no excitation in the ensembles $n-1$ and $n$.

Now we would like to use this $\mathrm{W}$ class state in one of the communication protocols. Imagine that we need to spread an entangled state between atomic ensembles with unknown coefficients to more than one parties. We choose a three-party protocol by way of example and it will become evident that there are many users that will work equally well.

Suppose there are three parties, the sender Alice, the receivers Bob and Carol. Alice entangles the ensembles 1, 2 and $3(4,5$ and 6$)$ in the $\mathrm{W}$ class state $|W\rangle_{123}\left(|W\rangle_{456}\right)$. The pair of ensembles $i$ and $i+3$ ( $i$ from 1 to 3 ) are put in the same place so that the ensembles $1,2,3$ and 4 , 5, 6 can be connected through the same optical channel, which fixed the phase shifts to be the same. So the states $|W\rangle_{123}$ and $|W\rangle_{456}$ can be shown using Eq. (7), where $\phi_{13}=\phi_{46}$ and $\phi_{12}=\phi_{45}$. The ensembles 2 and 5 are sent to Bob, 3 and 6 to Carol, 1 and 4 are left for herself.
Alice wants to teleport an atomic "polarization" state $|\varphi\rangle_{\text {un }}=\left(\alpha s_{L}^{+}+\beta s_{R}^{+}\right)|v a c\rangle$ [22], with unknown coefficients $\alpha$ and $\beta,|\alpha|^{2}+|\beta|^{2}=1$. Then she connects the ensembles $L$ and $1, R$ and 4 by manipulating the repumping lase pulses with frequency $\omega_{\text {repump }}$ on them synchronistically (shown in Fig 3). If the ensemble is in the metastable state after the repumping pulse, the transition $|e\rangle \rightarrow|s\rangle$ will occur determinately. The forwardscattering Stokes pulses are interfered at the beam splitters after the filters. If Alice get two clicks, one in D1 or D2, and the other in D3 or D4, the process is finished and the state of the ensembles of Bob and Carol is shown as

$$
\left[e^{i \phi_{13}}\left(\alpha s_{3}^{+}+\beta s_{6}^{+}\right)+e^{i \phi_{12}}\left(\alpha s_{2}^{+}+\beta s_{5}^{+}\right)\right]|v a c\rangle_{2356} \text {. }
$$

Otherwise, they should prepare the $\mathrm{W}$ class states and repeat the above steps until there are two correct clicks. Thus the state is teleported to the two receivers, either one of which equally likely receives the transmitted state, and similar to the scheme shown in Ref. [22], the teleportation fidelity would be nearly perfect.

It may be worth mentioning that if Bob and Carol perform a measurement, then one of them can recover the state with unit fidelity in a probabilistic manner. For example, Carol measures her ensembles 3 and 6 using two repumping laser pulses (seeing Fig 3 for details), and the Stokes pulses are collected by the detectors D5 and D6. If she obtains the original state $|\varphi\rangle_{\text {un }}$, there will be one click in either of the two detectors. Else, one excitation is registered from each ensembles, the original state is obtained by the other receiver Bob.

Finally, we have a brief conclusion. In this report, we describe an experimental scheme of entangling many atomic ensembles in the $\mathrm{W}$ class of maximally entangled states through laser manipulation. This protocol fits well the status of the current experimental technology. In addition, we show one of the applications of the kind of W class states, teleporting an entangled state between atomic ensembles with unknown coefficients to two distant parties, either one of which equally likely receives the transmitted state.

We thank L.-M. Duan for helpful discussion and Y.-S. Zhang and Z.-W. Zhou for their stimulating comments on an earlier version. This work was funded by $\mathrm{Na}$ tional Fundamental Research Program (2001CB309300), National Natural Science Foundation of China, the Innovation funds from Chinese Academy of Sciences, and also by the outstanding Ph. D thesis award and the CAS's talented scientist award entitled to Luming Duan.

[1] P. W. Shor, in Proceedings of the 35th Annual Sympo- 
sium on the Foundations of Computer Science, p. 124133 (IEEE Computer Society Press, Los Alamitos, California,1984).

[2] L. K. Grover, Phys. Rev. Lett. 79, 325 (1997).

[3] A. K. Ekert and R. Jozsa, Rev. Mod. Phys. 68, 733 (1996).

[4] C. H. Bennett et al., Phys. Rev. Lett. 70, 1895 (1993).

[5] D. Bouwmeester et al., Nature (London) 390, 575 (1997).

[6] C. H. Bennett and S. J. Wiesner, Phys. Rev. Lett. 69, 2881 (1992).

[7] A. K. Ekert, Phys. Rev. Lett. 67, 661 (1991).

[8] P. Xue, C.-F. Li, and G.-C. Guo, Phys. Rev. A, 64, 032305 (2001).

[9] P. Xue, C.-F. Li, and G.-C. Guo, Phys. Rev. A, 65, 034302 (2002).

[10] P. G. Kwiat et al., Phys. Rev. Lett. 75, 4337 (1995).

[11] P. G. Kwiat, E. Waks, A. G. White, I. Appelbaum and P. H. Eberhard, Phys. Rev. A 60, R773 (1999).

[12] A. G. White, D. F. V. James, P. H. Eberhard and P. G. Kwiat, Phys. Rev. Lett. 83, 3103 (1999).

[13] E. Hagley et al., Phys. Rev. Lett. 79, 1 (1997); Q. A. Turchette et al., Phys. Rev. Lett. 81, 3631 (1998).

[14] D. Bouwmeester et al., Phys. Rev. Lett. 82, 1345 (1999).

[15] A. Rauschenbeutel et al., Science 288, 2024 (2000).

[16] C. A. Sackett et al., Nature (London) 404, 256 (2000).

[17] J. W. Pan et al., Phys. Rev. Lett. 86, 4435 (2001).

[18] D. Gottesman, and I. L. Chuang, Nature (London) 402, 390 (1999).

[19] M. A. Nielsen, and I. L. Chuang, Quantum Computation and Quantum Information, (Cambridge University Press, UK, 2000).

[20] M. D. Lukin et al., Phys. Rev. Lett. 87, 037901 (2001).

[21] L.-M. Duan, J. I. Cirac, P. Zoller, and E. S. Polzik, Phys. Rev. Lett. 85, 5643 (2000).

[22] L.-M. Duan, M. D. Lukin, J. I. Cirac, and P. Zoller, Nature (London) 414, 413 (2001).

[23] L.-M. Duan, Phys. Rev. Lett. 88, 170402 (2002).

[24] A. Kuzmich, N. P. Bigelow, and L. Mandel, Europhys. lett. A 42481 (1998); J. Hald, J. L. Sorensen, C. Schori, and E. S. Polzik, Phys. Rev. Lett. 83, 1319 (1999); A. Kuzmich, L. Mandel, and N. P. Bigelow, Phys. Rev. Lett. 85, 1594 (2000).

[25] B. Julsgard, A. Kozhekin, and E. S. Polzik, Nature (London) 413, 400 (2001).

[26] L.-M. Duan, J. I. Cirac, and P. Zoller, Preprint quantph/0205005.

[27] W. Dür, G. Vidal, and J. I. Cirac, Phys. Rev. A 62, 062314 (2000).

[28] D. F. Phillips et al., Phys. Rev. Lett. 86, 783 (2001).

[29] C. Liu, Z. Dutton, C. H. Behroozi, and L. V. Hau, Nature (London) 409, 490 (2001).

[30] J. F. Roch et al. Phys. Rev. Lett. 78, 634 (1997).

Figure captions:

Figure 1. The relevant type $\Lambda$-level structure of the alkali atoms in the ensembles. A pair of metastable lower states $|g\rangle$ and $|s\rangle$ can be achieved, for example, in hyperfine or Zeeman sublevels of electronic ground states, and $|e\rangle$ is the excited state.

Figure 2. Schematic setup for entangling three ensembles 1, 2 and 3 in the $\mathrm{W}$ class state. The two ensembles
1 and 2 are in the EPR state $|\psi\rangle_{12}$, and the ensemble 3 is prepared in the ground state $|g\rangle$. The ensembles 2 and 3 are illuminated by the synchronized pumping laser pulses and the forward-scattering Stokes pulses are collected after the filters. The shutter $\mathrm{S} 1$ is on, and to avoid to destroy the single-photon detectors the other shutters must be off. If there is a click in D1 or D2, we open S2 and apply a repumping laser pulse to the ensemble 2 . When we connect the ensembles 1 and 3 , the manipulation of $\mathrm{S} 3$ and $\mathrm{S} 4$ is as the same. The dashed line represents the pumping laser pulses with the transition frequency $\omega_{\text {pump }}$ and the solid line represents the Stokes pulses which come from the transition $|e\rangle \rightarrow|s\rangle$.

Figure 3. Schematic setup for the realization of quantum teleportation using the $\mathrm{W}$ class states. The two ensembles $L$ and $R$ are in an entangled state $|\varphi\rangle_{\text {un }}$, and the ensembles 1, 2 and 3 (4, 5 and 6$)$ are prepared in the $W$ class state $|W\rangle$. The solid line represents the repumping laser pulses with the transition frequency $\omega_{\text {repump }}$ and the dashed line represents the Stokes pulses which come from the transition $|e\rangle \rightarrow|g\rangle$. 


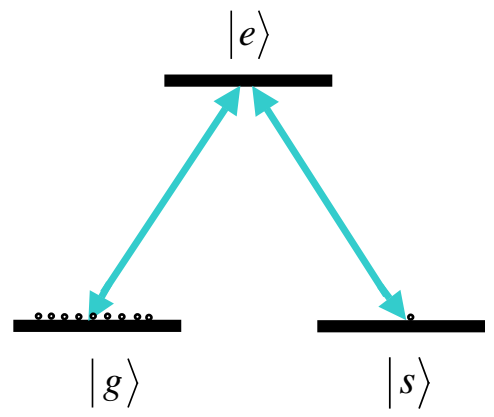

Figure. 1 


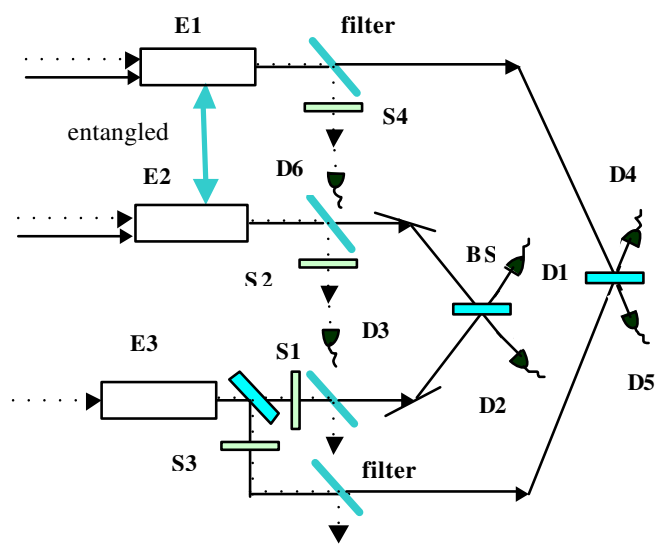

Figure. 2 


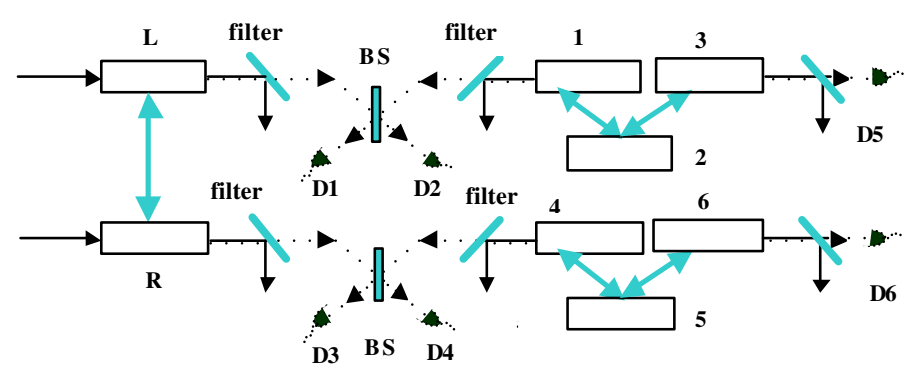

Figure. 3 